\title{
Fechner's paradox reflects a nonmonotone relation between binocular brightness and luminance
}

\author{
DWIGHT W. CURTIS and STANLEY J. RULE \\ University of Alberta, Edmonton, Alberta, Canada T6G 2E9
}

\begin{abstract}
Twenty subjects judged the brightness of binocularly fused targets whose monocular luminances were varied independently. On each trial, the left eye was presented with one of two relatively high luminances and the right eye was presented with one of 15 luminances from the range in which Fechner's paradox is effective. The objective of the experiment was to determine whether the psychophysical function over this range was nonmonotonic and U-shaped, as implied by several models of binocular brightness, or monotone increasing, but discontinuous at zero right-eye luminance. The functions associated with both left-eye intensities were found to be nonmonotone. Both minima were near the upper bound of the mesopic range.
\end{abstract}

Fechner's paradox refers to a phenomenon in binocular vision that is found when the intensity of a luminous target is markedly different for the two eyes. The phenomenon may be observed by fixating a target with both eyes and interposing a neutral density filter between one eye, say the right, and the target. If the right eye is subsequently closed, brightness will appear to increase. This is considered paradoxical because the total stimulation is greater in the binocular viewing condition, while brightness is greater for the monocular view.

The implications of Fechner's paradox for an understanding of how binocular information concerning brightness is processed is not entirely clear. Some early investigators (e.g., Fry \& Bartley, 1933; Robinson, 1895) assumed that it represented only a part of the total picture. When the luminances presented to the two eyes were more nearly equal, these investigators argued, binocular brightness exceeded the brightness of either monocular view, indicating partial binocular summation. But some more recent investigators attributed the summation effect to an artifact of the brightness matching procedures used in its observation (Engel, 1969; Levelt, 1965). In particular, they argued that the effects that appeared to be a consequence of summation were due to peripheral events associated with contour and contrast between the test and comparison targets, and proposed that in the absence of such interstimulus interactions, binocular brightness represented a compromise, or

This research was supported by Research Grants A9582 and A0151 from the Natural Sciences and Engineering Research Council of Canada. Requests for reprints should be sent to Dwight W. Curtis, Department of Psychology, University of Alberta, Edmonton, Alberta T6G 2E9. Canada. average, of the outputs of the two monocular channels. Consistent with their interpretation is the observation that under those viewing conditions that yield Fechner's paradox, the brightness of the dichoptically viewed target falls between the brightnesses of the two corresponding monocular views.

In a recent paper, we presented the results of experiments in which subjects were required to directly estimate the brightness of dichoptic targets in which the left-eye and right-eye luminances were selected from a factorial design. Direct scaling was used, instead of the matching paradigm employed in most previous studies of binocular brightness, because it enabled us to avoid any possibility that judgments of brightness were influenced by lateral interactions within monocular visual fields. In the more usual investigations of binocular brightness, a dichoptically fused test target at one location in the visual field was matched by a second target that was simultaneously in the field, so that the intensity setting of the matching stimulus might reflect lateral interactions (e.g., lateral inhibition) between it and the test stimulus. Engel (1970) has demonstrated that the magnitude of the apparent summation obtained in a matching experiment is inversely related to the separation of the test and matching targets. In our experiments, subjects evaluated the brightness of the test targets presented alone, thus avoiding any possible intervention of interactions. Magnitude estimations and category ratings each provided clear evidence for binocular compromise in brightness when the luminances presented to the two eyes were disparate, and partial binocular summation when the luminances were more nearly equal. These results were in conflict with the prediction of averaging models, such as those of Engel (1969) and Levelt 
(1965), for which apparent summation would be expected only when the monocular stimuli implicated lateral interactions. One averaging model, the centroid model (De Weert \& Levelt, 1974), a variant of models proposed earlier by MacLeod (1972) and Schrödinger (1926), predicts some summation in the absence of lateral interaction, but not of the magnitude that we obtained.

The mathematical description of our data required an expession that included a term for an interaction between the monoptic components, in addition to separate components associated with inputs to each eye. The trends in the data, together with certain mathematical considerations, suggested a family of functions that provide a satisfactory description of the relation between binocular and monocular brightness. A simple member of the family is given by the expression

$$
\psi_{\mathrm{ij}}=\left(\psi_{\mathrm{Li}^{2}}+\psi_{\mathrm{Rj}}{ }^{2}-\mathrm{b} \psi_{\mathrm{Li}} \psi_{\mathrm{Rj}}\right)^{1 / 2},
$$

in which $\psi_{\mathrm{ij}}$ denotes binocular brightness, and $\psi_{\mathrm{Li}}$ and $\psi_{R j}$ represent the brightness of the left- and right-eye targets when they are viewed monoptically. By letting $\mathrm{b}=2 \cos \alpha$, Equation 1 may be interpreted geometrically as the sum of two vectors with magnitudes $\psi \mathrm{i}$ and $\psi \mathrm{j}$, separated by an angle of $\alpha$ degrees. And when $90 \mathrm{deg}<\alpha<120 \mathrm{deg}$, Equation 1 predicts both that binocular brightness should represent a compromise between the monocular brightnesses when the luminances presented to the two eyes are grossly different and that it should exceed either monocular brightness when their luminances approach equality. Curtis and Rule (1978) found estimates of $\alpha$ and of the exponent of the psychophysical power function from three separate experiments to be in very close agreement.

The present paper is concerned with the form of the binocular brightness function predicted by the vector-sum model over the range of stimulation in which Fechner's paradox is observed. If one eye is presented with a constant high luminance and the intensity presented to the other eye is increased from zero luminance, the phenomenon itself implies that brightness should vary according to either of two trends. One possibility is that brightness changes as a continuous and nonmonotone function of the varied luminance such that at low intensities the function is decreasing, while at higher intensities it is increasing. Both Fechner and Aubert reported the function to be of this form (see Robinson, 1895, for a review of the early literature). This is also the form of the function predicted by the vector-sum and some weighted-averaging models. An alternative and less paradoxical possibility is that the function is discontinuous at a zero level of the variable target (i.e., monocular viewing) and monotone increasing for all nonzero intensities (i.e., binocular viewing). De Weert and Levelt's (1974) results from an analysis of binocular brightness judgments in terms of a simple additive model seems to imply the latter function.

Although the data are sparse, those that have been obtained appear to favor the nonmonotone relation; however, the studies that have been conducted have included few stimuli that were sufficiently low in intensity for a thorough examination of the initial trend. The strongest evidence has been provided by Fry and Bartley's (1933) experiments using brightness matching. When their targets were presented against a dark background, one point on the matching function was found to be on the decreasing portion of the curve. The number of points on this part of the curve was increased to three when the targets were presented against a low-intensity contrasting background. It should be noted, however, that some features of Fry and Bartley's data are peculiar. In particular, in matching a binocular target, in which the luminances to the two eyes were equal, to a second binocular target, also with equal luminances to the two eyes, the luminance settings should have been approximately equal. Instead, the luminance of the matching stimulus was set consistently well below that of the test stimulus. The source of this discrepancy is unknown. Engel (1969) has suggested that it may have been due to a calibration error.

The present study was designed to examine this portion of the function more thoroughly by obtaining a greater number of points in the lower region.

\section{METHOD}

The stimuli were 3-deg binocularly fused targets, produced by projecting the beams of two Kodak random-access projectors onto milk glass diffusers that were masked to produce 2 -cm circular targets. The targets were viewed through $2-\mathrm{mm}$ artificial pupils, and prisms were adjusted for each observer so that the monocular targets were fused by the relaxed eyes. The light sources were attenuated by Kodak Wratten neutral density filters mounted in slide holders. The relation was studied for two luminances presented to the left eye, 1,838 and $3,210 \mathrm{~cd} \mathrm{~m}^{-2}$. Relatively high intensities were employed because our previous research indicated that, if the relation was nonmonotone, the minimum should occur when the relative intensities to the two eyes was a small fraction. The presentation of a high luminance to the left eye permitted a sufficient number of low intensities to be presented to the right eye to map the trend of the initial portion of the function. Fifteen luminances were presented to the right eye: 0 , $.078, .378, .755,1.753,3.968,6.870,11.85,18.97,32.60,65.64$, $153.6,250.4,531.4$, and $796.6 \mathrm{~cd} \mathrm{~m}^{-2}$. Luminances were measured by a Spectra Spotmeter, Model VBD $-1^{\circ}$, from the viewpoint of the observer. The sets of luminances to the right and left eyes were crossed to generate 30 binocular combinations. Twenty undergraduate and graduate students served as paid observers. Each was first dark adapted and then presented with four replications of the stimulus series, each in a random permutation. The stimuli were presented for $.7 \mathrm{sec}$, with an interstimulus interval of $25 \mathrm{sec}$.

Direct judgments of brightness were employed to avoid conditions for lateral interactions between stimuli presented in the 
same monocular field as in a matching procedure. A category method was used in which subjects rated brightness on a 7-point scale. Results of our previous study (Curtis \& Rule, 1978) had indicated that category ratings and magnitude estimates yielded the same ordinal trend for judgments of binocularly fused targets.

\section{RESULTS AND DISCUSSION}

The first replication for each subject was treated as a practice series. The data analyzed were the median ratings from the remaining three replications for the 20 observers. Medians were employed rather than, say, arithmetic means because distributions of category ratings tend to be positively skewed for stimuli assigned lower category values and negatively skewed for stimuli toward the upper end of the scale. In the present instance, scale values based on means and medians exhibited the same trend, except for a slight flattening of the mean function for the most intense right-eye luminances, apparently caused by a ceiling effect.

The median ratings are plotted in Figure 1 as a function of right-eye luminance. The spacing along the abscissa represents luminance raised to the onethird power, which permits both a representation of zero luminance and an expansion of the scale toward the origin of the physical measure. The semi-interquartile range associated with each point was computed as a measure of dispersion. The mean and standard deviation of these measures were .74 and .20 , respectively. Plots of the first and third quartiles of subjects' ratings revealed the same trends as those for the medians plotted in Figure 1.

Fechner's paradox can be observed in the trends by comparing brightness for monoptic viewing, which corresponds to zero luminance to the right-eye, with the brightness for dichopic conditions for the lower, but nonzero, right-eye luminances. Some evidence for partial summation at the higher luminances is also present, which is consistent with our previous findings (Curtis \& Rule, 1978).

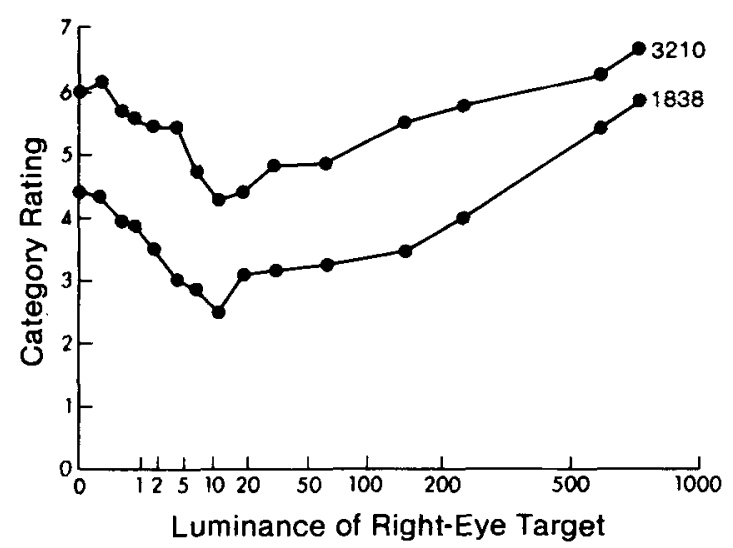

Figure 1. Binocular brightness as a function of right-eye luminance $\left(\mathrm{cd} \mathrm{m}^{-2}\right)$. The parameter is left-eye luminance.
Of primary interest is the finding that, for binocular viewing, the curves are nonmonotone. At the lower intensities, apparent brightness decreases as luminance to the right eye increases. The initial negative slope would seem to implicate an interocular inhibitory mechanism that, for a range of low intensities, produces increased inhibition with increased luminance. Fry and Bartley's (1933) hypothesis was that the mechanisms in each eye subserving brightness contrast tend to inhibit information concerning brightness from the opposing eye, and that binocular brightness constituted the algebraic sum of the excitatory and inhibitory activities from the two eyes.

A feature of the data from the present experiment that may be theoretically significant in explicating an inhibitory mechanism is that maximum interference occurred when each of the two left-eye luminances was combined with a right-eye luminance of $11.85 \mathrm{~cd}$ $\mathrm{m}^{-2}$. All right-eye stimuli below that associated with each minimum are from the mesopic range, in which both rod and cone systems are effective. The upper limit of the range is usually considered to be in the neighborhood of $10 \mathrm{~cd} \mathrm{~m}^{-2}$ (Le Grand, 1975). There is considerable evidence that the rod and cone systems interact (e.g., Makous \& Bothe, 1974; McCann, 1972; Whitten \& Brown, 1973); however, only monocular rod-cone interactions have been considered previously, and these have appeared to be retinal in origin. The present results suggest the possibility that, up to the extreme of the mesopic range, rod activity in one eye exerts an increasing inhibitory influence upon cone activity in the other eye. If so, the locus of the interaction should be at a higher level, possibly at the lateral geniculate, but more likely at the cortical level. This would yield the decreasing portion of the brightness function, while the positive relation found at higher luminances is explicable on the assumption that a summing mechanism operates to combine photopic brightness information from the two eyes.

\section{REFERENCES}

Curtis, D. W., \& Rule, S. J. Binocular processing of brightness information: A vector-sum model. Journal of Experimental Psychology: Perception and Performance, 1978, 4, 132-143.

De Weent, C. M. M., \& Levelt, W. J. M. Binocular brightness combinations: Additive and nonadditive aspects. Perception \& Psychophysics, 1974, 15, 551-562.

ENGEL, G. R. The auto-correlation function and binocular brightness mixing. Vision Research, 1969, 9, 1111-1130.

ENGEL, G. R. Tests of a model of binocular brightness. Canadian Journal of Psychology, 1970, 24, 335-352.

Fry, G. A., \& Bartley, S. H. The brilliance of an object seen binocularly. American Journal of Ophthalmology, 1933, 16, 687-693.

Le Grand, Y, Measurement of the visual stimulus. In E. C. Carterette \& M. P. Friedman (Eds.), Handbook of perception (Vol. V) Seeing, New York: Academic Press, 1975.

Lfifit, W. J. M. Binocular brightness averaging and contour information. British Journal of Psychology, 1965, 56, 1-13. 
MacLeoD, D. I. A. The Schrödinger equation in binocular brightness combination. Perception, 1972, 1, 321-324.

Makous, N., \& Bothe, R. Cones block signals from rods. Vision Research, 1974, 14, 285-294.

McCANN, J. J. Rod-cone interactions: Different color sensations from identical stimuli. Science, 1972, 176, 1255-1257.

Robinson, T. R. Experiments on Fechner's paradox. American Journal of Psychology, 1895, 7, 9-25.
Schrödinger, E. Muller-Pouillets Lehrbuch der Physik. 11. Auflage, Zweiter Band, Braunschweig: Vieweg, 1926.

Whitten, D. N., \& Brown, K. T. Photopic suppression on monkey's rod receptor potential, apparently by a cone-initiated lateral inhibition. Vision Research, 1973, 13, 1629-1658.

(Received for publication September 3, 1979; revision accepted December $10,1979$. 